\title{
On the (non)existence of symplectic resolutions of linear quotients
}

\author{
Gwyn Bellamy and Travis Schedler
}

\begin{abstract}
We study the existence of symplectic resolutions of quotient singularities $V / G$, where $V$ is a symplectic vector space and $G$ acts symplectically. Namely, we classify the symplectically irreducible and imprimitive groups, excluding those of the form $K \rtimes S_{2}$ where $K<$ $\mathrm{SL}_{2}(\mathbf{C})$, for which the corresponding quotient singularity admits a projective symplectic resolution. As a consequence, for $\operatorname{dim} V \neq 4$, we classify all symplectically irreducible quotient singularities $V / G$ admitting a projective symplectic resolution, except for at most four explicit singularities, that occur in dimensions at most 10, for which the question of existence remains open.
\end{abstract}

\section{Introduction}

Symplectic quotient singularities have been intensively studied over the past decade, due to their rich geometric structures, as illustrated by the symplectic McKay correspondence [5], and their key role in the geometric representation theory of symplectic reflection algebras. One of the key questions regarding the geometry of symplectic quotient singularities is whether there exist symplectic resolutions of the singularity. In this paper, we classify all irreducible quotient singularities of dimension not equal to four which admit a projective symplectic resolution, excluding four exceptional cases. The main step is to prove that, in dimension four, a large class of singularities do not admit a resolution.

More precisely, we classify all symplectically imprimitive and irreducible symplectic reflection groups (excluding the groups $K \rtimes S_{2}<\mathrm{Sp}_{4}(\mathbf{C})$ where $K<\mathrm{SL}_{2}(\mathbf{C})$ ) whose corresponding quotient singularity admits a projective symplectic resolution. This is an important step in an ongoing program to

2010 Mathematics Subject Classification: 16S80, 17B63.

Key words and phrases: symplectic resolution, symplectic smoothing, symplectic reflection algebra, Poisson variety, quotient singularity, McKay correspondence. 
completely classify all finite subgroups $G$ of $\operatorname{Sp}(V)$ such that $V / G$ admits a symplectic resolution.

In order to state our main result we introduce some notation. Let $(V, \omega)$ be a symplectic vector space and $G \subset \operatorname{Sp}(V)$ a finite group. We are interested in the singularities of the quotient $V / G$. In particular, the quotient $V / G$ is said to admit a (projective) symplectic resolution if there exists a (projective) resolution of singularities $\pi: X \rightarrow V / G$ such that $X$ is a symplectic manifold; see section 4.2 for the precise definition.

The number of known examples of such symplectic quotient singularities admitting symplectic resolutions is remarkably small: they are only products of the following singularities:

- The infinite series $\mathbf{C}^{2 n} /\left(K \succ S_{n}\right)$, where $K$ is a finite subgroup of $S L_{2}(\mathbf{C})$ (here and below, $K \backslash S_{n}:=K^{n} \rtimes S_{n}$ ), and

- Two exceptional quotients $\mathbf{C}^{4} / G$ : the exceptional complex reflection group $G_{4}<\mathrm{GL}_{2}(\mathbf{C})<\operatorname{Sp}_{4}(\mathbf{C})[2,15]$, and the group $Q_{8} \times \mathbf{z} / 2 D_{8}<$ $\mathrm{Sp}_{4}(\mathbf{C})[3]$.

We will assume throughout that $V$ is a symplectically irreducible representation of $G$, i.e., that $V$ does not admit a proper nonzero symplectic vector subspace invariant under $G$. As we will recall, all quotients that admit a symplectic resolution are products of singularities $V / G$ of this form.

As a consequence of our main result we prove:

Theorem 1.1. Let $(V, G)$ be symplectically irreducible. If $V / G$ admits a projective symplectic resolution and $\operatorname{dim} V \neq 4$ then $(V, G) \simeq\left(\mathbf{C}^{2 n}, K\right.$ ? $\left.S_{n}\right)$ for $K<\mathrm{SL}_{2}(\mathbf{C})$, unless possibly $(V, G)$ is one of four examples.

The four examples referred to above, whose corresponding reflection representation $V$ has dimension at most 10, will be clarified below, and in these cases we do not resolve the question of whether $V / G$ admits a projective symplectic resolution.

To state our main theorem, we introduce some more definitions. Recall that an element $g \in G$ is said to be a symplectic reflection if $\operatorname{rk}(1-g)=2$. The group $G$ (or rather the triple $(V, \omega, G)$ ) is said to be a symplectic reflection group if $G$ is generated by the symplectic reflections that it contains. By [18], if $V / G$ admits a projective symplectic resolution, then $G$ is a symplectic reflection group.

If $V$ were not symplectically irreducible, then $V$ would decompose as $V=V_{1} \oplus V_{2}$ where $V_{i}$ are symplectic representations of $G$. When $G$ is a symplectic reflection group as above, then $G$ must decompose as $G=G_{1} \times G_{2}$ 
where $G_{i}<\operatorname{Sp}\left(V_{i}\right)$ is generated by symplectic reflections in $\operatorname{Sp}\left(V_{i}\right)$; hence $V / G=V_{1} / G_{1} \times V_{2} / G_{2}$. Therefore the classification of quotients $V / G$ admitting symplectic resolutions reduces to the case where $V$ is symplectically irreducible.

A symplectic representation $V$ of $G$ is said to be symplectically imprimitive if there exists a nontrivial decomposition $V=V_{1} \oplus \cdots \oplus V_{k}$ into symplectic subspaces such that, for all $i$ and all $g \in G$, there exists $j$ such that $g\left(V_{i}\right)=V_{j}$. We call a group $G<\mathrm{Sp}(V)$ symplectically irreducible, resp. symplectically imprimitive, if $V$ is such as a representation of $G$.

As above, let $K$ be a finite subgroup of $S L_{2}(\mathbf{C})$ (the classification of such groups is well known; see $\S 3$ below). The wreath product $K$ ? $S_{n}$ acts as a symplectic reflection group on $\mathbf{C}^{2 n}$. By [7, Theorem 2.2 and 2.9], the symplectically imprimitive and irreducible symplectic reflection groups are all realized as subgroups (normal when $n>2$ ) of $K<S_{n}$, for suitable $K$ and $n$.

When $n=2$, we will exclude the subgroups $K \rtimes S_{2}<(K \times K) \rtimes S_{2}$, where $K \hookrightarrow(K \times K)$ is given by $k \mapsto(k, \alpha(k))$ for some involution $\alpha: K \rightarrow$ $K$. Our main result reads:

Theorem 1.2. Let $G<\mathrm{Sp}_{2 n}(\mathbf{C})$ be symplectically imprimitive and irreducible. Assume that either $n>2$ or that $G$ is not of the form $K \rtimes S_{2}$ as above with $K<\mathrm{SL}_{2}(\mathbf{C})$. Then the symplectic quotient $\mathbf{C}^{2 n} / G$ admits a projective symplectic resolution if and only if $G$ is isomorphic to either $K$ ? $S_{n}$ or $Q_{8} \times_{\mathbf{Z} / 2} D_{8}$, which is the group from [3] (for which $n=2$ ).

In more detail, by [7, Theorem 2.2 and 2.9], the symplectically imprimitive and irreducible symplectic reflection groups $G<\mathrm{Sp}_{2 n}(\mathbf{C})$ are, up to conjugation, all of the form:

- For $n=2$, the group $G=G(K, H, \alpha)$, where $H<K$ is a normal subgroup, $\alpha$ is an involution of $K / H$, and $G(K, H, \alpha)<K \backslash S_{2}$ is the subgroup generated by $S_{2}, H^{2}$, and the cosets $(k H, \alpha(k H))<K^{2} / H^{2}$ for all $k \in K$;

- For $n \geq 3$, the group $G=G_{n}(K, H)$, where $H<K$ is a subgroup containing the commutator subgroup $[K, K]$, and $G_{n}(K, H)<K$ 乙 $S_{n}$ is generated by $S_{n}, H^{n}$, and the cosets $\left(k_{1} H, \ldots, k_{n} H\right)<K^{n} / H^{n}$ for all $k_{1}, \ldots, k_{n} \in K$ such that $k_{1} \cdots k_{n} \in H$.

Using results of Kaledin, we will reduce the theorem to the case $n=2$, together with the single case $G_{3}\left(\mathrm{D}_{2}, \mathrm{C}_{2}\right)$. The condition that $G(K, H, \alpha)$ is not the group $K \rtimes S_{2}$ is precisely saying that $H$ is nontrivial. Therefore the 
main step of the proof is to show that the groups $G:=G(K, H, \alpha)$ do not admit projective symplectic resolutions when $H \neq\{1\}$. Let $\mathrm{Y}_{H}$ denote the minimal resolution of $\mathbf{C}^{2} / H$. The key to proving Theorem 1.2 is to study the action of the quotient $G / H^{2}$ on $\mathrm{Y}_{H} \times \mathrm{Y}_{H}$. In particular, we show that the symplectic variety $\left(\mathrm{Y}_{H} \times \mathrm{Y}_{H}\right) /\left(G / H^{2}\right)$ does not, in general, admit a projective symplectic resolution.

\section{1.}

The existence of projective symplectic resolutions of the quotient singularity $V / G$ is known to be equivalent to the existence of a smooth Poisson deformation of $V / G$; that is, a flat, affine Poisson deformation of $V / G$ whose generic fibre is a smooth Poisson variety. Let $\mathrm{H}_{\mathbf{c}}(G)$ be the symplectic reflection algebra at $t=0$ associated to $G$ as defined in [9]. The centre of this algebra is denoted $\mathbf{Z}_{\mathbf{c}}(G)$. When the parameter $\mathbf{c}$ is zero, $\mathbf{Z}_{\mathbf{c}}(G)$ is the coordinate ring of $V / G$. It is known, by [11], that $\mathbf{Z}_{\mathbf{c}}(G)$ defines a flat Poisson deformation of $V / G$. As noted in [3, Theorem 1.2.1], results of Ginzburg-Kaledin and Namikawa imply that:

Corollary 1.3. Let $G$ be a symplectically imprimitive symplectic reflection group obeying the assumption of Theorem 1.2. Then the variety $\operatorname{Spec} Z_{\mathbf{c}}(G)$ is singular for all parameters $\mathbf{c}$ unless $G$ is isomorphic to $K$ ? $S_{n}$ or $Q_{8} \times \mathbf{Z} / 2$ $D_{8}$.

Usually one uses the representation theory of symplectic reflection algebras to show that the variety $\operatorname{Spec} \mathbf{Z}_{\mathbf{c}}(G)$ is singular for all parameters and hence deduce that the corresponding symplectic quotient singularity does not admit a projective symplectic resolution. We have taken the opposite approach in this paper.

\section{2 .}

The paper is structured as follows. In section two we recall the definition of symplectic variety and symplectic resolutions. Using work of Namikawa and Kaledin we give two general criteria for the non-existence of projective symplectic resolutions of $V / G$. In section three, in order to fix notation, we recall the Kleinian groups. Cohen's classification of symplectic reflection groups is recalled in section four.

In section five we consider more specific criteria that can be used to prove the non-existence of projective symplectic resolutions of $V / G$ when 
$G$ is symplectically imprimitive and $V=\mathbf{C}^{4}$. Then, in section six, we work through the list of such groups, showing case-by-case that they do not posses projective symplectic resolutions. In section seven, we deduce the main result for $\operatorname{dim} V \geq 6$ from these cases and one additional case, in Lemma 7.1. In section eight, we summarize the resulting proof of Theorem 1.2, and deduce Theorem 1.1 from this. Finally, in section nine, we list some open questions ("exercises for the interested reader").

\section{Symplectic varieties and symplectic resolutions}

\section{1.}

In this section we recall the definition of a symplectic variety and of symplectic resolutions. We give some criteria for the (non-)existence of projective symplectic resolutions. The definition of symplectic variety was introduced by Beauville in the seminal paper [1].

Definition 2.1. Let $X$ be an affine variety. Then $X$ is said to be a symplectic variety if

1) $X$ is normal.

2) There exists a symplectic form $\omega$ on the smooth locus $X_{\mathrm{sm}}$ of $X$.

3) There exists a resolution of singularities $\pi: Y \rightarrow X$ such that $\pi^{*} \omega$ extends to a regular 2-form on $Y$.

One says that $X$ admits a symplectic resolution if there is a resolution of singularities $\pi: Y \rightarrow X$ such that $\pi^{*} \omega$ extends to a non-degenerate 2 -form on $Y$.

\subsection{Conic symplectic varieties}

Let $X$ be an affine symplectic variety. Then $X$ is said to be equipped with a good $\mathbf{C}^{\times}$-action if there is an algebraic action of $\mathbf{C}^{\times}$on $X$ such that

1) The weights of $\mathbf{C}^{\times}$on $X$ are positive and there exists a unique fixed point $0 \in X$.

2) The symplectic form $\omega$ has positive weight $l>0$.

Let $X$ and $Y$ be normal, quasi-projective varieties over $\mathbf{C}$ and $\Omega_{X}$, resp. $\Omega_{Y}$ the corresponding canonical sheaves. A morphism $\pi: Y \rightarrow X$ is said to 
be crepant if $\pi^{*} \Omega_{X} \simeq \Omega_{Y}$. If $X$ is a symplectic variety, $Y$ a smooth variety and $\pi: Y \rightarrow X$ a crepant, proper birational morphism, then $\pi$ is a symplectic resolution. The composition of two crepant morphisms is again crepant. The following is a direct consequence of the results of [17].

Theorem 2.2. Let $X$ be an affine symplectic variety equipped with a good $\mathbf{C}^{\times}$-action. Let $\pi: Y \rightarrow X$ be a crepant, projective, birational morphism and let $U \subset Y$ be an affine open subset. If $X$ admits a projective symplectic resolution then $U$ admits a projective symplectic resolution.

Proof. Assume that $X$ admits a projective symplectic resolution. Since $X$ is equipped with a good $\mathbf{C}^{\times}$-action, the proof of [17, Theorem 5.5] shows that every crepant, projective, birational morphism $\pi_{0}: Z_{0} \rightarrow X$ from a space $Z_{0}$ having at worst $\mathbf{Q}$-factorial terminal singularities is necessarily a symplectic resolution. By [6], the minimal model program implies that there exists some crepant, projective, birational morphism $\rho: Z \rightarrow Y$ such that $Z$ has only Q-factorial terminal singularities. Therefore $\pi \circ \rho: Z \rightarrow X$ must be a projective symplectic resolution, by the first observation. In particular, $Z$ is a symplectic manifold. The restriction $\left.\rho\right|_{\rho^{-1}(U)}: \rho^{-1}(U) \rightarrow U$ is a resolution of singularities. Since $\rho^{-1}(U)$ is a symplectic manifold, [10, Proposition 1.6] implies that $\left.\rho\right|_{\rho^{-1}(U)}$ is a projective symplectic resolution of $U$.

\section{3.}

The case that will be of interest to us is the following. Let $(V, \omega, G)$ be a symplectic reflection group acting on a symplectic vector space $V$ and assume that we are given a normal subgroup $H$ of $G$ such that

- $H$ acts on $V$ as a symplectic reflection group.

- There exists a projective symplectic resolution $\pi: X \rightarrow V / H$.

- The action of $Q:=G / H$ lifts to an action on $X$ making $\pi$ a $G / H$ equivariant morphism.

To each $x \in X$, we associate the pair $\left(Q_{x}, T_{x} X\right)$, where $Q_{x}$ is the stabilizer of $x$ in $Q$ and $T_{x} X$ is the tangent space of $X$ at $x$. Note that $T_{x} X$ is a symplectic representation of $Q_{x}$. This representation is faithful. To see this we note that the fact that $\pi$ is equivariant implies that $Q$ acts freely on some dense open subset of $X$. On the other hand, if there is some $g \in Q_{x}$ acting trivially on $T_{x} X$, then this implies that $\operatorname{dim} \operatorname{Fix}_{X}(g)=\operatorname{dim} X$ and hence $g=1$. 
Proposition 2.3. If the quotient $V / G$ admits a projective symplectic resolution then $T_{x} X / Q_{x}$ admits a projective symplectic resolution for all $x \in X$.

Proof. Let $\mathbf{C}^{\times}$act on $V$ with weight one. This action commutes with the action of $G$ and the symplectic form $\omega$ has weight 2 with respect to this action. Therefore, it descends to a good $\mathbf{C}^{\times}$-action on $V / G$. Hence, as in Theorem 2.2, we may choose some crepant projective morphism $\rho: Y \rightarrow$ $X / Q$ such that $Y$ has at worst $\mathbf{Q}$-factorial, terminal singularities. As shown there, the fact that there exists some projective symplectic resolution of $V / G$ implies that $Y$ is smooth. Choose $x \in X$ and let $\bar{x}$ denote its image in $X / Q$. Write $\widehat{Y}_{x}$ for the completion of $Y$ along the closed sub-scheme $\rho^{-1}(\bar{x})$. The completion of $X / Q$ at $\bar{x}$ is isomorphic to the quotient of the completion $\widehat{T_{x} X}$ by $Q_{x}$. Then $\rho$ induces a projective morphism $\widehat{\rho}: \widehat{Y}_{x} \rightarrow \widehat{T_{x} X} / Q_{x}$. Since $\widehat{Y}_{x}$ is smooth with trivial canonical bundle, $\widehat{\rho}$ is a projective smooth formal crepant resolution in the sense of $[14, \S 1]$. Therefore [14, Theorem 1.4] implies that $T_{x} X / Q_{x}$ admits a projective symplectic resolution.

\section{The Kleinian groups}

\section{1 .}

Let $\mathbf{H}=\mathbf{R} \oplus \mathbf{R i} \oplus \mathbf{R j} \oplus \mathbf{R} \mathbf{k}$ be the skew-field of quaternions. In order to fix notation, we remark that the finite subgroups of $\mathrm{GL}\left(\mathbf{H}^{1}\right)$ up to isomorphism are the cyclic group $C_{m}=\left\langle\boldsymbol{\zeta} \mid \boldsymbol{\zeta}^{m}=1\right\rangle$, of order $m$, the binary dihedral group $\mathrm{D}_{m}=\left\langle\mathrm{C}_{2 m}, \mathbf{k}\right\rangle$, of order $4 m$ and the three exceptional groups $\mathrm{T}=$ $\left\langle\mathrm{D}_{2}, \boldsymbol{\omega}\right\rangle$ of order 24 , where $\boldsymbol{\omega}=\frac{1}{2}(1-\mathbf{i}+\mathbf{j}+\mathbf{k}), \mathrm{O}=\left\langle\mathrm{T}, \frac{1}{\sqrt{2}}(1+\mathbf{i})\right\rangle$ of order 48 and

$$
\begin{aligned}
& \mathrm{I}=\left\langle\mathrm{D}_{2}, \frac{1}{2}(\rho+\sigma \mathbf{i}-\mathbf{j})\right\rangle, \text { of order } 120, \\
& \qquad \text { where } \rho=2 \cos \left(\frac{\pi}{5}\right) \text { and } \sigma=2 \cos \left(\frac{3 \pi}{5}\right) .
\end{aligned}
$$

\subsection{Complexification}

We consider $\mathbf{C} \subseteq \mathbf{H}$ to be the subfield $\mathbf{C}=\mathbf{R} \oplus \mathbf{R} \cdot \mathbf{i}$. Given a finite subgroup $G$ of $\mathrm{GL}\left(\mathbf{H}^{n}\right)$, a choice of complex structure on $\mathbf{H}$ realizes $G$ as a subgroup of $\mathrm{GL}\left(\mathbf{C}^{2 n}\right.$ ) (we consider matrices acting on the right of $\mathbf{H}^{n}$ ). The standard choice of complex structure, as used in [7] is $\mathbf{H}=\mathbf{C} \oplus \mathbf{C j}$. However, in order to use the results of [13], we choose the complexification $\mathbf{H}=\mathbf{C} \oplus \mathbf{C k}$. The procedure $G \subset \mathrm{GL}\left(\mathbf{H}^{n}\right)$ goes to $G^{\vee} \subset \mathrm{GL}\left(\mathbf{C}^{2 n}\right)$ is called 
complexification. Noting that $\epsilon:=e^{\frac{2 \pi i}{8}}=\frac{1+i}{\sqrt{2}}$, complexification is uniquely defined by

$$
\mathbf{i}=\left(\begin{array}{cc}
i & 0 \\
0 & -i
\end{array}\right), \quad \mathbf{j}=\left(\begin{array}{cc}
0 & -i \\
-i & 0
\end{array}\right), \quad \mathbf{k}=\left(\begin{array}{cc}
0 & 1 \\
-1 & 0
\end{array}\right)
$$

The complexification map defines an embedding $\mathrm{GL}\left(\mathbf{H}^{n}\right) \hookrightarrow \mathrm{Sp}\left(\mathbf{C}^{2 n}\right)$, where $\mathbf{H}$ is viewed as a two-dimensional symplectic complex vector space with the form $\langle 1, \mathbf{k}\rangle=1$. As explained in $[7, \S 1]$, this induces an equivalence between finite subgroups of $\mathrm{GL}\left(\mathbf{H}^{n}\right)$, up to conjugation, and finite subgroups of $\mathrm{Sp}\left(\mathbf{C}^{2 n}\right)$, up to conjugation.

This explicitly realizes all the finite subgroups of $\mathrm{GL}(\mathbf{H})$ above as subgroups of $\mathrm{Sp}_{2}(\mathbf{C})=\mathrm{SL}_{2}(\mathbf{C})$. Moreover, this explicitly realizes the subgroups of [7] as subgroups of $\mathrm{Sp}_{2 n}(\mathbf{C})$, since they are described there in terms finite subgroups of $\mathrm{GL}\left(\mathbf{H}^{n}\right)$.

\section{Cohen's classification of symplectic reflection groups}

\section{1 .}

The irreducible symplectic reflection groups were first classified by A. Cohen in [7]. We recall here the the outline of his classification. His results are stated in terms of quaternionic reflection groups, but, as explained in section 3 , the results of $[7, \S 1]$ show that there is a bijective correspondence between quaternionic and symplectic reflection groups preserving symplectic imprimitivity and symplectic irreducibility.

A symplectic reflection group $G<\mathrm{Sp}(V)$ is said to be improper if it preserves a Lagrangian subspace $L \subseteq V$, so that $G<\mathrm{GL}(L)$ is a complex reflection group and $V \simeq L \oplus L^{*}$ as a $G$-module (otherwise, we say that $G$ is proper). Complex reflection groups were classified by Chevalley, Shephard, and Todd, and for these groups our main results are immediate consequences of [2]. Thus from now on we assume $G$ is proper.

We further say that a symplectic reflection group is complex imprimitive if it is imprimitive considered as a subgroup of $\mathrm{GL}\left(\mathbf{C}^{2 n}\right)$, i.e., if there exists a decomposition $\mathbf{C}^{2 n}=V_{1} \oplus \cdots \oplus V_{k}$ into complex subspaces such that, for all $g \in G$ and all $i$, there exists $j$ such that $g\left(V_{i}\right)=V_{j}$. There exist symplectically primitive symplectic reflection groups which are, nonetheless, complex imprimitive. Therefore there are three natural classes to consider (assuming, without loss of generality that $G$ is symplectically irreducible and proper): 
1) The symplectically imprimitive symplectic reflection groups. These are the groups that we will consider in this paper. By [7, Theorem 2.2], such subgroups of $\mathrm{Sp}_{4}(\mathbf{C})$ are of the form $G(K, H, \alpha)$ as defined in the introduction, and by [7, Theorem 2.6], they must be listed in Tables 1 and 2. By [7, Theorem 2.9], such subgroups of $\operatorname{Sp}_{2 n}(\mathbf{C})$ for $n>2$ are of the form $G_{n}(K, H)$, as defined in the introduction. Conversely, all of these groups are symplectically imprimitive (and irreducible) symplectic reflection groups.

2) The symplectically primitive symplectic reflection groups which are complex imprimitive. These all lie in $\mathrm{Sp}_{4}(\mathbf{C})$ and are classified in $[7$, Lemma 3.3]. The fact that those listed are all such groups follows from [7, Theorem 3.6].

3) The complex primitive symplectic reflection groups. In this case, $[7$, Theorem 4.2] say that the groups in this case are precisely those listed in [7, Table III]. Thus there are only thirteen such groups and they occur in dimension at most ten.

It is interesting to note that the three exceptional groups $P_{1}, P_{2}$, and $P_{3}$ that occur in (3) above are all extensions of some group by the symplectic reflection group $Q_{8} \times \mathbf{z} / 2 D_{8}$ studied in [3].

\subsection{Symplectically imprimitive groups}

We denote by $K$ a finite subgroup of $S L_{2}(\mathbf{C})$ and $H$ a normal subgroup of $K$. The Kleinian singularity $\mathbf{C}^{2} / H$ is denoted $\mathbf{X}_{H}$ and the corresponding minimal resolution is $\pi: \mathrm{Y}_{H} \rightarrow \mathrm{X}_{H}$.

We let $\alpha$ be an involution of $\Gamma:=K / H$. We choose coordinates on $\mathbf{C}^{2}$ so that the ring of polynomial functions on $\mathbf{C}^{2}$ is $\mathbf{C}[x, y]$. We also endow $\mathbf{C}^{2}$ with the standard symplectic form so that $\{x, y\}=1$, where $\{-,-\}$ is the corresponding Poisson bracket. Associated to $H, K, \alpha$ is the symplectically imprimitive and irreducible group $G:=G(K, H, \alpha)$, acting on $\mathbf{C}^{4}=\mathbf{C}^{2} \times$ $\mathbf{C}^{2}$. The explicit description of $G$ as a subgroup of $K$ ? $S_{2}$ was given in the introduction. In $\S 4$ below, we recall the action of $G$ and $K$ ? $S_{2}$ on $\mathbf{C}^{4}$.

Recall also that the pair (id, $\alpha$ ) defines an embedding of $K$ into $K^{2}$. This induces an embedding $K / H$ into $(K / H)^{2}$. Since $\alpha$ has order at most $2, S_{2}$ preserves the image of this map and we may form a twisted semidirect product $(K / H) \rtimes_{\alpha} S_{2}$. Then $G / H^{2} \simeq(K / H) \rtimes_{\alpha} S_{2}$. The involutions in $(K / H) \rtimes_{\alpha} S_{2}$ are all given by $\left\{(x, \alpha(x)) \mid x^{2}=1\right\} \cup\left\{(x, \alpha(x)) \cdot s_{12} \mid\right.$ $x \alpha(x)=1\}$. The group $G / H^{2}$ is denoted $Q$. 
If $T$ is a group, $\rho$ a representation of $T$ and $g$ an automorphism of $T$ then the twist of $\rho$ by $g$ is denoted ${ }^{g} \rho$. As a vector space, ${ }^{g} \rho=\rho$ and $t \cdot m:=g(t) m$ for $t \in T$ and $m \in{ }^{g} \rho$.

\section{3.}

We continue to take $G=G(K, H, \alpha)$. Let $s_{12} \in S_{2}<G<K<S_{2}$ be the transposition. Following [7, $§ 2]$, let $L_{\alpha}:=\{x \in K \mid x \alpha(x) \in H\}$. As observed in [7], the symplectic reflections in $G$ are the elements of $H \times\{1\},\{1\} \times H$, and $\left\{\left(x, x^{-1}\right) s_{12} \mid x \in L_{\alpha}\right\}$.

Lemma 4.1. The subgroup $H<S_{2}$ is normal in $G$ if and only if $\alpha=1$, and the subgroup $H^{2}$ is always normal in $G$.

Proof. By considering $k=(x, y) \cdot s_{12}$ acting by conjugation on $H^{2} \cdot s_{12}$, where $(x, y) \in K^{2}$ satisfies $y \in \alpha(x H)$, we see that $H<S_{2}$ is normal in $G$ if and only if $x H y^{-1}=H$ for all $x \in K$ and $y \in \alpha(x H)$. But this is the same as saying that $\alpha=1$ on the quotient. The second statement is clear.

\section{4 .}

A complete list of groups $G(K, H, \alpha)$ (up to isomorphism) is given in Table 1 . In Table 1 the automorphism $\alpha_{r} \in \operatorname{Aut}\left(\mathbf{D}_{m}\right)$ is defined by $\alpha_{r}(u)=$ $u^{r} ; \alpha_{r}(v)=v$, where $\mathbf{D}_{m}=\left\langle u, v \mid u^{m}=v^{2}=(u v)^{2}=1\right\rangle$ and the automorphism $\beta_{r} \in \operatorname{Aut}\left(\mathrm{D}_{m}\right)$ is defined by $\beta_{r}\left(\boldsymbol{\zeta}_{m}\right)=\boldsymbol{\zeta}_{m}^{r}$ and $\beta_{r}(\mathbf{k})=-\mathbf{k}$.

\section{Singular subgroups of $G$}

This section is rather technical, therefore we provide an outline. We wish to show that the groups $G(K, H, \alpha)$, with $H \neq\{1\}$, do not admit symplectic resolutions. The purpose of this section is to provide two general criteria, Theorem 5.4 and Proposition 5.6, for the non-existence of projective symplectic reflections for these groups. This is done by analyzing the set of points on $\mathrm{Y}_{H} \times \mathrm{Y}_{H}$ that have non-trivial stabilizer under the action of the group $Q$ and showing that this set has components of dimension zero.

\section{1 .}

A subgroup $P$ of $\Gamma$ is said to be a parabolic subgroup if there exists some $x \in \mathrm{Y}_{H}$ such that $\operatorname{Stab}_{\Gamma}(x)=P$. The set of all points $x$ in $\mathrm{Y}_{H}$ such that 
Table 1: Nonconjugate Symplectically Imprimitive, Symplectically Irreducible Proper Four-Dimensional Reflection Groups $G(K, H, \alpha)$

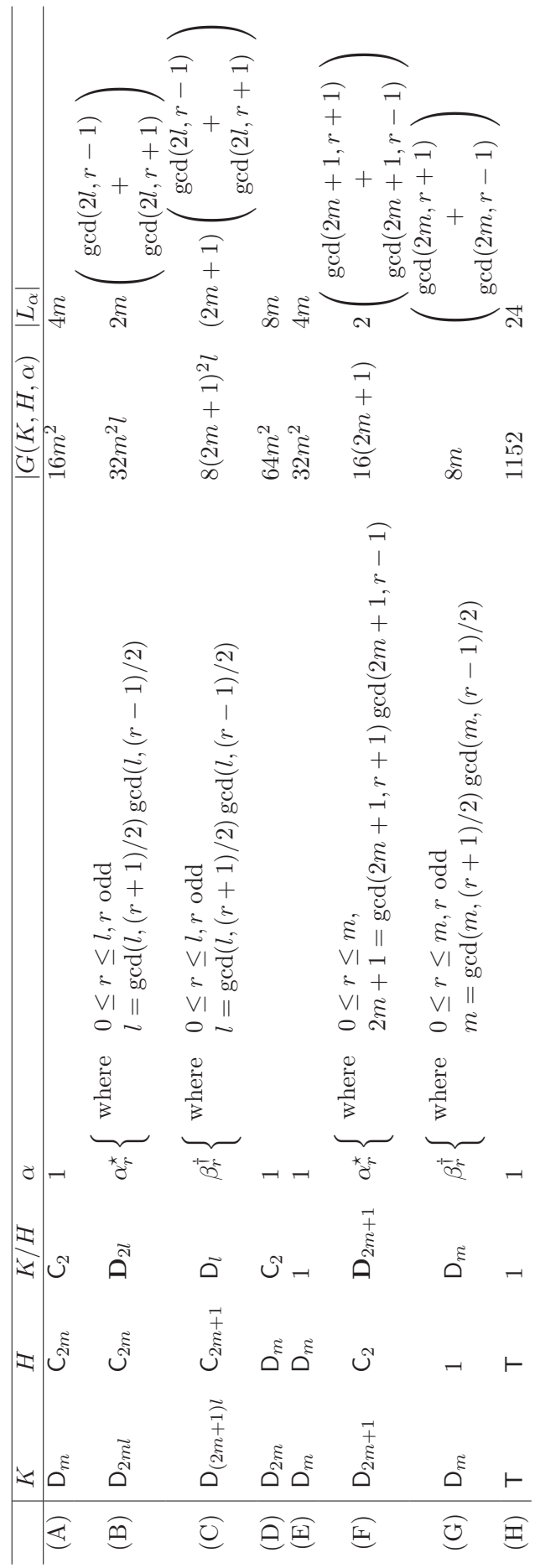


Table 2: Nonconjugate Symplectically Imprimitive, Symplectically Irreducible Proper Four-Dimensional Reflection Groups $G(K, H, \alpha)$ (cont.)

\begin{tabular}{l|llllll}
\hline & $K$ & $H$ & $K / H$ & $\alpha$ & $\mid G(K, H, \alpha)$ & $\left|L_{\alpha}\right|$ \\
\hline$(\mathrm{I})$ & $\mathrm{T}$ & $\mathrm{D}_{2}$ & $\mathrm{C}_{3}$ & Inversion & 384 & 24 \\
$(\mathrm{~J})$ & $\mathrm{T}$ & $\mathrm{C}_{2}$ & $\mathrm{Alt}(4)$ & Conjugation by $(12)$ & 96 & 12 \\
$(\mathrm{~K})$ & $\mathrm{T}$ & 1 & $\mathrm{~T}$ & Conjugation by $(i-j)$ & 48 & 12 \\
$(\mathrm{~L})$ & $\mathrm{O}$ & $\mathrm{O}$ & 1 & 1 & 4608 & 48 \\
$(\mathrm{M})$ & $\mathrm{O}$ & $\mathrm{T}$ & $\mathrm{C}_{2}$ & 1 & 2304 & 48 \\
$(\mathrm{~N})$ & $\mathrm{O}$ & $\mathrm{D}_{2}$ & $\mathrm{D}_{3}$ & 1 & 768 & 32 \\
$(\mathrm{O})$ & $\mathrm{O}$ & $\mathrm{C}_{2}$ & $\mathrm{Sym}(4)$ & 1 & 192 & 14 \\
$(\mathrm{P})$ & $\mathrm{O}$ & 1 & $\mathrm{O}$ & Conjugation by $k$ & 96 & 18 \\
$(\mathrm{Q})$ & $\mathrm{O}$ & 1 & $\mathrm{O}$ & $1 \neq \alpha \in$ Ker $:$ Aut(O) $\rightarrow$ Aut(Sym(4)) & 96 & 14 \\
$(\mathrm{R})$ & $\mathrm{I}$ & $\mathrm{I}$ & 1 & 1 & 14400 & 120 \\
$(\mathrm{~S})$ & $\mathrm{I}$ & $\mathrm{C}_{2}$ & $\mathrm{Alt}(5)$ & 1 & 480 & 32 \\
$(\mathrm{~T})$ & $\mathrm{I}$ & $\mathrm{C}_{2}$ & $\mathrm{Alt}(5)$ & Conjugation by $(12)$ & 480 & 20 \\
$(\mathrm{U})$ & $\mathrm{I}$ & 1 & $\mathrm{I}$ & Conjugation by $j$ & 240 & 30 \\
$(\mathrm{~V})$ & $\mathrm{I}$ & 1 & $\mathrm{I}$ & Preimage of conjugation by $(12)$ & 240 & 20 \\
& & & under Aut(I) $\rightarrow$ Aut(Alt(5)) & & \\
\hline
\end{tabular}

$\operatorname{Stab}_{\Gamma}(x)=P$ is denoted $\mathrm{Y}_{H}(P)$. Since $\mathrm{Y}_{H}$ is smooth, $\mathrm{Y}_{H}(P)$ is a smooth, though not generally connected, locally closed subset of $Y_{H}$. We denote by $\mathrm{Y}_{H}(P)_{0}$, resp. $\mathrm{Y}_{H}(P)_{>0}$, the union of all components of dimension zero, resp. greater than zero, in $\mathrm{Y}_{H}(P)$. Key to our classification theorem is the following technical definition.

Definition 5.1. Let $P$ be a parabolic subgroup of $\Gamma$.

1) A point $x \in \mathrm{Y}_{H}(P)_{0}$ is isolated if there exists no parabolic subgroup $\{1\} \neq T \subsetneq P$ such that $x \in \overline{Y_{H}(T)}$.

2) The group $P$ is said to be singular if there exist isolated points $x \in$ $\mathrm{Y}_{H}(P)_{0}$ and $y \in \mathrm{Y}_{H}(\alpha(P))_{0}$ such that $y \notin \Gamma \cdot x$.

Note that a singular subgroup of $\Gamma$ is actually the data of a four-tuple $(P, x ; \alpha(P), y)$.

Lemma 5.2. If $P$ is a singular subgroup of $\Gamma$ then there exists an affine open, Q-stable subset $U \subset \mathrm{Y}_{H} \times \mathrm{Y}_{H}$ and closed point $p \in U$ such that $\operatorname{Stab}_{Q}(p)=P$ and $Q$ acts freely on $U \backslash Q \cdot p$, where $P$ is realized as a subgroup of $Q$ via (id, $\alpha)$.

Proof. Let $P=P_{1}, P_{2}=\alpha(P), \ldots, P_{k}$ be all the conjugates of either $P$ or $\alpha(P)$ in $\Gamma$. The dense open subset of $\mathrm{Y}_{H} \times \mathrm{Y}_{H}$ consisting of all points with 
trivial stabilizer is denoted $\left(\mathrm{Y}_{H} \times \mathrm{Y}_{H}\right)_{\text {reg }}$ and we write $\mathrm{Y}_{H}\left(P_{i}\right)_{\text {iso }}$ for the set of all isolated points in $\mathrm{Y}_{H}\left(P_{i}\right)_{0}$. Set

$$
U_{0}=\left(\mathrm{Y}_{H} \times \mathrm{Y}_{H}\right)_{\mathrm{reg}} \cup\left[\bigcup_{i=1}^{k} \mathrm{Y}_{H}\left(P_{i}\right)_{\text {iso }} \times \mathrm{Y}_{H}\left(\alpha\left(P_{i}\right)\right)_{\text {iso }}\right]
$$

It is a $Q$-stable, dense subset of $\mathrm{Y}_{H} \times \mathrm{Y}_{H}$. Let

$$
\Delta=\bigcup_{h \in \Gamma}\left\{(h \cdot x, x) \in \mathrm{Y}_{H} \times \mathrm{Y}_{H} \mid x \in \mathrm{Y}_{H}\right\} .
$$

It is a proper, closed, $Q$-stable subvariety of $\mathrm{Y}_{H} \times \mathrm{Y}_{H}$. We set $U_{1}=U_{0} \backslash \Delta$.

Claim 5.3. $U_{1}$ is a $Q$-stable, dense open subset of $\mathrm{Y}_{H} \times \mathrm{Y}_{H}$.

Proof. That $U_{1}$ is $Q$-stable and dense is straight-forward. To see that it is open, we note firstly that the stabilizer of any $x \in U_{1}$ is either trivial or $($ id $\times \alpha)\left(P_{i}\right)$. This follows from the fact that if $s_{12} \cdot(h, \alpha(h))$ stabilizes $x$ for some $h \in \Gamma$, then $x \in \Delta$. Now decompose

$$
\left(\mathrm{Y}_{H} \times \mathrm{Y}_{H}\right) \backslash U_{1}=\bigsqcup_{\beta} C_{\beta}
$$

into the connected components of the stabilizer stratification of $\left(\mathrm{Y}_{H} \times \mathrm{Y}_{H}\right) \backslash$ $U_{1}$. To show that $U_{1}$ is open, it suffices to show that $\bar{C}_{\beta} \cap U_{1}=\emptyset$ : clearly $\bar{C}_{\beta} \cap\left(\mathrm{Y}_{H} \times \mathrm{Y}_{H}\right)_{\mathrm{reg}}=\emptyset$, therefore if $\bar{C}_{\beta} \cap U_{1} \neq \emptyset$, then there exists some point $x \in \bar{C}_{\beta} \backslash C_{\beta} \cap U_{1}$ whose stabilizer is $(\mathrm{id} \times \alpha)\left(P_{i}\right)$. If $y \in C_{\beta}$ then $\operatorname{Stab}_{Q}(y) \subseteq(\mathrm{id} \times \alpha)\left(P_{i}\right)$, which implies that $y=\left(y_{1}, y_{2}\right)$ with $\operatorname{Stab}_{\Gamma}\left(y_{1}\right) \subseteq$ $P_{i}$. In this case there exists some connected components $D_{1}$ of $Y_{H}\left(\operatorname{Stab}_{\Gamma}\left(y_{1}\right)\right)$ and $D_{2}$ of $\mathrm{Y}_{H}\left(\alpha\left(\operatorname{Stab}_{\Gamma}\left(y_{1}\right)\right)\right)$ such that $C_{\beta} \subset D_{1} \times D_{2}$ is a dense subset. This implies that $x \in \overline{D_{1} \times D_{2}}$, contradicting the fact that $x=\left(x_{0}, x_{1}\right)$ with each $x_{i}$ isolated. This completes the proof of the claim.

By assumption, there exists some $x \in \mathrm{Y}_{H}(P)_{0}$ and $y \in \mathrm{Y}_{H}(\alpha(P))_{0}$ such that $y \notin \Gamma \cdot x$. Set $p=(x, y)$. The condition $y \notin \Gamma \cdot x$ implies that $p \notin \Delta$. Therefore it belongs to $U_{1}$. We may choose an open subset $U_{2}$ of $p$ in $U_{1}$ such that the stabilizer of every point in $U_{2}$ (except $p$ itself) has trivial stabilizer. Then the set $U$ we require is $\bigcup_{g \in Q} g \cdot U_{2}$, except that $U$ may not be affine. However, [4, Lemma 1.3] shows that we can replace $U$ by a smaller, affine open and $Q$-stable neighborhood of $p$.

Theorem 5.4. If there exists a singular subgroup $P$ of $\Gamma$ then $V / G$ does not admit a projective symplectic resolution. 
Proof. Let

$$
F=\bigcup_{1 \neq h \in Q} \operatorname{Fix}(h)
$$

be the closed subset of $\mathrm{Y}_{H} \times \mathrm{Y}_{H}$ consisting of all points with non-trivial stabilizer. If $U$ is the open subset of $\mathrm{Y}_{H} \times \mathrm{Y}_{H}$ whose existence is guaranteed by Lemma 5.2 , then $U \cap F=Q \cdot p$ is a finite union of points. Let $x \in U \cap F$ and $Q_{x}$ the stabilizer of $x$. By assumption $Q_{x} \neq 1$. By assumption, $Q_{x}$ acts freely on $T_{x} U \backslash\{0\}$, therefore $T_{x} U / Q_{x}$ is an isolated symplectic singularity. Hence $Q_{x}$ contains no symplectic reflections. Therefore $\left(T_{x} U, Q_{x}\right)$ does not admit any symplectic resolution by Verbitsky's Theorem, [18]. Then Proposition 2.3 implies that $V / G$ does not admit a projective symplectic resolution.

\section{2 .}

The case where $H=\mathrm{C}_{2}$ occurs several times in Tables 1 and 2 . In this case $\mathrm{Y}_{H}=T^{*} \mathbf{P}^{1}$ and the action of $\Gamma$ on $T^{*} \mathbf{P}^{1}$ comes from the embedding $\Gamma \hookrightarrow P S L_{2}(\mathbf{C})=\operatorname{Aut}\left(\mathbf{P}^{1}\right)$. Let $V=\mathbf{C}^{2}$ with basis $v_{1}, v_{2}$ and $x_{1}, x_{2}$ the dual basis of $V^{*}$ so that $\mathbf{C}[V]=\mathbf{C}\left[x_{1}, x_{2}\right], \mathbf{C}\left[V^{*}\right]=\mathbf{C}\left[v_{1}, v_{2}\right]$. Then

$$
T^{*} \mathbf{P}^{1}=\left\{(v, w) \in(V \backslash\{0\}) \times V^{*} \mid w(v)=0\right\} / \mathbf{C}^{\times} .
$$

The charts $U_{1}=\left(x_{2} \neq 0\right), U_{2}=\left(x_{1} \neq 0\right)$ cover $T^{*} \mathbf{P}^{1}$ and

$$
\mathbf{C}\left[\pi^{-1}\left(U_{1}\right)\right]=\mathbf{C}\left[\frac{x_{1}}{x_{2}}, v_{1} x_{2}\right], \quad \mathbf{C}\left[\pi^{-1}\left(U_{2}\right)\right]=\mathbf{C}\left[\frac{x_{2}}{x_{1}}, v_{2} x_{1}\right]
$$

where $\pi: T^{*} \mathbf{P}^{1} \rightarrow \mathbf{P}^{1}$. We say that $T \subset \Gamma$ is a maximal cyclic subgroup of $\Gamma$ if it is a cyclic subgroup of $\Gamma$ and there is no other cyclic subgroup of $\Gamma$ that properly contains $T$.

Lemma 5.5. Let $p \in \mathbf{P}^{1}$ and $T=\operatorname{Stab}_{\Gamma}(p)$. Then $T$ is a maximal cyclic subgroup of $\Gamma$.

Proof. Assume that $1 \neq g \in \Gamma$ fixes $p$. Diagonalizing $g$, we may assume that $p=[1: 0]$ or $[0: 1]$. This implies that every non-zero $g$ fixes exactly two 
points in $\mathbf{P}^{1}$. If $h$ is another non-zero element of $\Gamma$ that fixes $[1: 0]$, then

$$
g=\left(\begin{array}{cc}
\eta & 0 \\
0 & \eta^{-1}
\end{array}\right), \quad h=\left(\begin{array}{cc}
\zeta & b \\
0 & \zeta^{-1}
\end{array}\right)
$$

where $\eta, \zeta$ are some roots of unity. The matrix $h$ above has infinite order if $b \neq 0$. Therefore the fact that $\Gamma$ is finite implies that $g$ and $h$ are contained in some common cyclic subgroup of $\Gamma$ and fix the same points of $\mathbf{P}^{1}$. Hence the stabilizer of $[1: 0]$ in $\mathbf{P}^{1}$ is some maximal cyclic subgroup of $\Gamma$.

Proposition 5.6. If there exists a point $p \in \mathbf{P}^{1}$ such that $\left|\operatorname{Stab}_{\Gamma}(p)\right|>2$, then there exists a point $x \in T^{*} \mathbf{P}^{1} \times T^{*} \mathbf{P}^{1}$ such that $\left(Q_{x}, T_{x}\left(T^{*} \mathbf{P}^{1} \times T^{*} \mathbf{P}^{1}\right)\right)$ does not admit a symplectic resolution.

Proof. By Lemma 5.5, the stabilizer of $p$ is a maximal cyclic subgroup of $\Gamma$. Therefore we may assume that

$$
\operatorname{Stab}_{\Gamma}(p)=\langle s\rangle \simeq C_{m}
$$

for some $m>2$. Let $r=\alpha(s)$. Thinking of $s$ and $r$ as elements of order $2 m$ in $K$, we may assume that $s$ is a diagonal matrix with respect to the basis $v_{1}, v_{2}$ of $V$. We choose another basis $w_{1}, w_{2}$ of $V$ and dual basis $y_{1}, y_{2}$ of $V^{*}$ such that $r$ is diagonal with respect to these basis. Then there exists a primitive $2 m^{\text {th }}$ root of unity $\zeta$ such that

$$
s \cdot v_{1}=\zeta v_{1}, \quad s \cdot v_{2}=\zeta^{-1} v_{2}, \quad r \cdot w_{1}=\zeta^{a} w_{1}, \quad r \cdot w_{2}=\zeta^{-a} w_{2},
$$

for some integer $a$ coprime to $2 m$. Let $p_{1}=[1: 0], p_{2}=[0: 1]$, resp. $q_{1}=[1$ : $0], q_{2}=[0: 1]$, with respect to the coordinates $\left[x_{1}: x_{2}\right]$, resp. $\left[y_{1}: y_{2}\right]$ of $\mathbf{P}^{1}$. Then $s \cdot p_{i}=p_{i}$ and $r \cdot q_{i}=q_{i}$ for $i=1,2$. Let $\mathbf{x}=\left(p_{1}, q_{1}\right) \in T^{*} \mathbf{P}^{1} \times T^{*} \mathbf{P}^{1}$. Recall that $Q=(K / H) \rtimes_{\alpha} S_{2}$. It is a subgroup of $(K / H) \curlywedge S_{2}=(K / H)^{2} \cup$ $(K / H)^{2} \cdot s_{12}$. We decompose $T=\operatorname{Stab}_{Q}(\mathbf{x})$ as

$$
T_{0}=T \cap(K / H)^{2}, \quad T_{1}=(K / H)^{2} \cdot s_{12}
$$

There are two cases to consider: case $a) T_{1}=\emptyset$, and case $\left.b\right) T_{1} \neq \emptyset$.

We begin by considering case $a$ ). In this case we have $T=T_{0}=\left\langle s_{(1)} r_{(2)}\right\rangle$. Let $e_{1}, e_{2}, f_{1}, f_{2}=\left(\frac{x_{1}}{x_{2}}\right)^{*},\left(v_{1} x_{2}\right)^{*},\left(\frac{y_{1}}{y_{2}}\right)^{*},\left(w_{1} y_{2}\right)^{*}$ so that

$$
\left.T_{\mathbf{x}}\left(T^{*} \mathbf{P}^{1} \times T^{*} \mathbf{P}^{1}\right)\right)=\mathbf{C} \cdot\left\{e_{1}, e_{2}, f_{1}, f_{2}\right\}
$$


With respect to this chosen basis of $T_{\mathbf{x}}\left(T^{*} \mathbf{P}^{1} \times T^{*} \mathbf{P}^{1}\right)$, we have

$$
s_{(1)} r_{(2)}=\left(\begin{array}{cccc}
\zeta^{2} & 0 & 0 & 0 \\
0 & \zeta^{-2} & 0 & 0 \\
0 & 0 & \zeta^{2 a} & 0 \\
0 & 0 & 0 & \zeta^{-2 a}
\end{array}\right)
$$

This is not a symplectic reflection. Therefore $\left(Q_{\mathbf{x}}, T_{\mathbf{x}}\left(T^{*} \mathbf{P}^{1} \times T^{*} \mathbf{P}^{1}\right)\right)$ does not admit a symplectic resolution by Verbitsky's Theorem, [18].

In the second case, there exists some $h \in \Gamma$ such that $t=s_{12} h_{(1)} \alpha(h)_{(2)} \in$ $T_{1}$ (in fact, $T_{1}=T_{0} \cdot t$ ). Hence $h \cdot p_{1}=q_{1}$ and $\alpha(h) \cdot q_{1}=p_{1}$. This implies that

$$
\alpha(h) h=s^{\lambda}, \quad h \alpha(h)=r^{\mu},
$$

for some $\lambda$ and $\mu$. Applying $\alpha$ to the above equations shows that in fact $\lambda=\mu$. Moreover

$$
h^{-1} \operatorname{Stab}_{\Gamma}\left(q_{1}\right) h=\alpha(h) \operatorname{Stab}_{\Gamma}\left(q_{1}\right) h=\operatorname{Stab}_{\Gamma}\left(p_{1}\right) .
$$

Therefore, $h \cdot p_{2}=q_{2}$ and $\alpha(h) \cdot q_{2}=p_{2}$. If $\operatorname{Stab}_{Q}(x)$ is generated by symplectic reflections then we may assume that $t$ is a symplectic reflection. We have

$$
t^{2}=\alpha(h)_{(1)} h_{(1)} h_{(2)} \alpha(h)_{(2)}=\left(s_{(1)} r_{(2)}\right)^{\lambda} .
$$

This cannot be a symplectic reflection. Therefore it must be the identity, $\lambda=0$, and $t^{2}= \pm$ id in $G$. Possibly after rescaling, we have $h \cdot x_{1}=y_{1}$ and $h \cdot x_{2}=y_{2}$. Thus, with respect to the basis $e_{1}, e_{2}, f_{1}, f_{2}$ above, $t$ is given by

$$
t=\left(\begin{array}{llll}
0 & 0 & 1 & 0 \\
0 & 0 & 0 & 1 \\
1 & 0 & 0 & 0 \\
0 & 1 & 0 & 0
\end{array}\right)
$$

Then $\operatorname{Stab}_{Q}(\mathbf{x})=C_{m} \cup C_{m} \cdot t$. The characteristic polynomial of $\left(s_{(1)} r_{(2)}\right)^{i} \cdot t$ is $u^{4}-\left(\zeta^{2 i(a+1)}+\zeta^{-2 i(a+1)}\right) u^{2}+1$. Therefore it is a symplectic reflection if and only if $\zeta^{2 i(a+1)}+\zeta^{-2 i(a+1)}=2$ i.e. $i(a+1)=0$ modulo $m$. If $a+1$ is not zero modulo $m$ then the solutions to this equation form a proper subgroup $C_{d}$ of $C_{m}$. Then the subgroup of $Q$ generated by all symplectic reflections is the proper subgroup $G(d, d, 2)$ of $Q$. Thus, for $\operatorname{Stab}_{Q}(\mathbf{x})$ to be a symplectic 
reflection group we must have $a=-1$. In this case,

$$
\left(\operatorname{Stab}_{Q}(x), T_{\mathbf{x}}\left(T^{*} \mathbf{P}^{1} \times T^{*} \mathbf{P}^{1}\right)\right) \simeq\left(G(m, m, 2), \mathfrak{h} \oplus \mathfrak{h}^{*}\right),
$$

where $\mathfrak{h}$ is the reflection representation for $G(m, m, 2)$. By [2], such a pair admits a symplectic resolution only if $m=1,2$.

Remark 5.7. Note that there will exist a point $p \in \mathbf{P}^{1}$ such that $\left|\operatorname{Stab}_{\Gamma}(p)\right|$ $>2$ if and only if there is an element in $K$ of order at least 6 .

\section{The action of $\Gamma$ on $\mathrm{Hilb}^{H}\left(\mathrm{C}^{2}\right)$}

In this section we show case-by-case, based on Tables 1 and 2, that the symplectically imprimitive and irreducible symplectic reflection groups $G(K, H, \alpha)$, with $H \neq\{1\}$, satisfy at least one of the criteria required to apply either Theorem 5.4 or Proposition 5.6. Our results are summarized in Theorem 6.4.

\section{1.}

The minimal resolution of $\mathbf{X}_{H}=\mathbf{C}^{2} / H$ is denoted $\mathbf{Y}_{H}$. We denote by Hilb ${ }^{n} \mathbf{C}^{2}$ the Hilbert scheme of $n$ points in the plane. This is a smooth, symplectic manifold of dimension $2 n$, see [16].

Proposition 6.1. The action of $G / H^{2}$ on $\mathrm{X}_{H} \times \mathrm{X}_{H}$ lifts to an action of $G / H^{2}$ on $\mathrm{Y}_{H} \times \mathrm{Y}_{H}$.

Proof. Let $n=|H|$. The action of $K$ on $\mathbf{C}^{2}$ induces an action of $K$ on Hilb $^{n} \mathbf{C}^{2}$. One can realize $\mathbf{Y}_{H}$ as the component Hilb ${ }^{H} \mathbf{C}^{2}$ of $\left(\text { Hilb }^{n} \mathbf{C}^{2}\right)^{H}$ whose generic point $I$ is a radical ideal (or in other words $V(I)$ is a free $H$-orbit). This is a $K$-stable subvariety of $\left(\operatorname{Hilb}^{n} \mathbf{C}^{2}\right)^{H}$ and $\mathrm{Y}_{H} \times \mathrm{Y}_{H}$ is a $G$-stable subvariety of Hilb ${ }^{n} \mathbf{C}^{2} \times$ Hilb $^{n} \mathbf{C}^{2}$. By definition, the action of $G$ on $\mathrm{Y}_{H} \times \mathrm{Y}_{H}$ factors through $G / H^{2}$.

\section{2 .}

We will identify $\mathrm{Y}_{H}$ with $\mathrm{Hilb}^{H} \mathbf{C}^{2}$ throughout this section. By the classical McKay correspondence, the vertices $\left\{\rho_{i}\right\}_{i \in I}$ of the Dynkin diagram can be put in bijection with the irreducible components of the exceptional fiber of the minimal resolution of $\mathbf{C}^{2} / H$ in such a way that the edges between two 
vertices are in bijection with the number of points (which is always 0 or 1 ) of intersection of the two irreducible components. The component labeled by the vertex $\rho_{i}$ is denoted $\mathbf{P}_{i}$. On the other hand, the vertices of the affine Dynkin diagram can be naturally labeled by the isomorphism classes $\operatorname{Irr}(H)$ of simple $H$-modules such that $\operatorname{dim} \operatorname{Hom}_{H}\left(\mathbf{C}^{2} \otimes \rho_{i}, \rho_{j}\right)$ is twice the number of edges between the vertices $\rho_{i}$ and $\rho_{j}$ (note that the representation $\mathbf{C}^{2}$ is self-dual so that $\left.\operatorname{dim} \operatorname{Hom}_{H}\left(\mathbf{C}^{2} \otimes \rho_{i}, \rho_{j}\right)=\operatorname{dim} \operatorname{Hom}_{H}\left(\mathbf{C}^{2} \otimes \rho_{j}, \rho_{i}\right)\right)$. The trivial representation $\rho_{0}$ labels an extending vertex of the affine diagram. Therefore $\operatorname{Irr}_{*}(H)=\operatorname{Irr}(H) \backslash\{$ triv $\}$ labels the vertices of the nonaffine Dynkin diagram. This allows us to define two actions of $\Gamma$ on the Dynkin diagram of $H$ by graph automorphisms. The geometric action is defined by $g \cdot \rho_{i}=\rho_{j}$ if $g\left(\mathbf{P}_{i}\right)=\mathbf{P}_{j}$ and the edge labeled by $p \in \mathbf{P}_{i_{1}} \cap \mathbf{P}_{i_{2}}$ is sent to the edge labeled by $g \cdot p \in g\left(\mathbf{P}_{i_{1}}\right) \cap g\left(\mathbf{P}_{i_{2}}\right)$. The representation action is defined by $g \cdot \rho_{i}=\rho_{j}$ if ${ }^{g} \rho_{i} \simeq \rho_{j}$ and if $g \cdot \rho_{i_{k}}=\rho_{j_{k}}$, for $k=1,2$ with $\operatorname{dim} \operatorname{Hom}_{H}\left(\mathbf{C}^{2} \otimes \rho_{i_{1}}, \rho_{i_{2}}\right)=2$ then the fact that $g \in S L_{2}(\mathbf{C})$ normalizes $H$ implies that ${ }^{g} \mathbf{C}^{2} \simeq \mathbf{C}^{2}$, hence

$$
\operatorname{dim} \operatorname{Hom}_{H}\left(\mathbf{C}^{2} \otimes \rho_{i_{1}}, \rho_{i_{2}}\right)=\operatorname{dim} \operatorname{Hom}_{H}\left(\mathbf{C}^{2} \otimes \rho_{j_{1}}, \rho_{j_{2}}\right)
$$

and so $g$ take the edge between $\rho_{i_{1}}$ and $\rho_{i_{2}}$ to the edge between $\rho_{j_{1}}$ and $\rho_{j_{2}}$. Using Hilb ${ }^{H} \mathbf{C}^{2}$, Ito and Nakumura, [13], constructed a natural bijection between the irreducible components of the exceptional fiber and $\operatorname{Irr}_{*}(H)$ in such a way that the geometric action and the representation action become equal (a beautiful case free proof was later given by Crawley-Boevey [8]). We recall their bijection. They showed that for $I \in \pi^{-1}(0)$, the socle of $\mathbf{C}[x, y] / I$ is either irreducible as a $H$-module or consists of a pair of non-isomorphic simple $H$-modules. Moreover, if $I \in \mathbf{P}_{i}$ does not belong to any other component then the socle of $\mathbf{C}[x, y] / I$ is irreducible and the isomorphism class of this simple module depends only on $\mathbf{P}_{i}$ (and not on the specific choice of $I)$. Hence we may label the Dynkin diagram so that the socle of $\mathbf{C}[x, y] / I$, with $I \in \mathbf{P}_{i}$ generic, is $\rho_{i}$. If $I \in \mathbf{P}_{i} \cap \mathbf{P}_{j}$ then they showed that

$$
\operatorname{soc}(\mathbf{C}[x, y] / I) \simeq \rho_{i} \oplus \rho_{j}, \quad \text { and } \quad \operatorname{dim}_{\operatorname{Hom}}\left(\mathbf{C}^{2} \otimes \rho_{i}, \rho_{j}\right)=2 .
$$

If the socle of $\mathbf{C}[x, y] / I$ is isomorphic to $\lambda$ say as a $H$-module then

Lemma 6.2. The bijection $\rho_{i} \leftrightarrow \mathbf{P}_{i}$ intertwines the geometric action and the representation action of $\Gamma$ on the Dynkin diagram of $H$.

Proof. It is straight-forward, but we include a brief explanation for the reader's convenience. Let $g \in K$. Applying $g$ to the short exact sequence 
$0 \rightarrow I \rightarrow \mathbf{C}[x, y] \rightarrow \mathbf{C}[x, y] / I \rightarrow 0$ and using the fact that ${ }^{g} \mathbf{C}[x, y] \simeq \mathbf{C}[x, y]$ as a $H$-module implies that ${ }^{g}(\mathbf{C}[x, y] / I) \simeq \mathbf{C}[x, y] / g(I)$. The identification restricts to ${ }^{g} \operatorname{Soc}(\mathbf{C}[x, y] / I) \simeq \operatorname{soc}(\mathbf{C}[x, y] / g(I))$.

The above lemma gives us an easy, representation theoretic way to describe the action of $\Gamma$ on the irreducible components of the exceptional fiber.

Remark 6.3. The action of $\Gamma$ on $Y_{H}$ preserves the symplectic form. Therefore, each component of the closed subvariety $\left(\mathrm{Y}_{H}\right)^{\Gamma}$ is either two-dimensional or zero-dimensional. Since $Y_{H}$ is irreducible and the action of $\Gamma$ is effective, we see that $\left(\mathrm{Y}_{H}\right)^{\Gamma}$ is actually a finite collection of points.

\section{3. (A)}

In this case we have $K=\mathrm{C}_{2 m}, H=\mathrm{D}_{m}$ and $\Gamma=\mathrm{C}_{2}$, which is generated by the image $g$ of $\mathbf{k}$. The irreducible representations of $C_{2 m}$ are labeled $\rho_{i}, 0 \leq$ $i \leq 2 m-1$ and ${ }^{g} \rho_{i}=\rho_{2 m-i}$. Therefore the only irreducible representations fixed by $\mathrm{C}_{2}$ are $\rho_{0}$ and $\rho_{m}$. As described above, the irreducible component of the exceptional locus corresponding to $\rho_{i}$ is denoted $\mathbf{P}_{i}$ so that $\mathbf{P}_{m}$ is the only component that is mapped to itself by $g$. There are exactly two points $p, q$ in $\mathbf{P}_{m}$ that are fixed $g$. Obviously, $q \notin \Gamma \cdot p$. Since $\alpha=1,\left(\mathrm{C}_{2}, p ; \mathrm{C}_{2}, q\right)$ is a singular subgroup of $\mathrm{C}_{2}$.

\section{4. (B)}

In this case we have $H=\mathrm{C}_{2 m}, K=\mathrm{D}_{2 m l}$ and $\Gamma=\mathbf{D}_{2 l}=\langle u, v\rangle$, where $u$ is the image of $\boldsymbol{\zeta}$ and $v$ is the image of $\mathbf{k}$. The element $u$ acts trivially on $\operatorname{Irr}\left(\mathrm{C}_{2 m}\right)$ and the action of $v$ is given by ${ }^{v} \rho_{i}=\rho_{2 m-i}$. Therefore $\operatorname{Stab}_{\mathbf{D}_{2 l}}\left(\rho_{i}\right)=$ $\mathrm{C}_{2 l}$ if $1 \leq i \neq m \leq 2 m-1$ and $\operatorname{Stab}_{\mathbf{D}_{2 l}}\left(\rho_{m}\right)=\mathbf{D}_{2 l}$. Provided $m \neq 1$, we choose some $1 \leq i \neq m \leq 2 m-1$ and let $p, q \in \mathbf{P}_{i}$ be the two points whose stabilizer is $\mathrm{C}_{2 l}=\langle u\rangle$. Since $v$ maps $\mathbf{P}_{i}$ to $\mathbf{P}_{2 m-i}$, we have $q \notin \Gamma \cdot p$. Noting that $\alpha_{r}\left(\mathrm{C}_{2 l}\right)=\mathrm{C}_{2 l},\left(\mathrm{C}_{2 l}, p ; \mathrm{C}_{2 l}, q\right)$ is a singular subgroup.

Assume now that $m=1$. Then we are in the situation described in (5.2). When $l=1$, the group $G$ is the subject of the paper [3], where it is shown that the corresponding quotient singularity admits a projective symplectic resolution. The group $\mathrm{D}_{2 l}$ contains a cyclic subgroup of order $4 l$. Therefore, when $l \geq 2$, there is an element in $K$ of order $\geq 8$. Therefore we may apply Proposition 5.6. 


\section{5. (C)}

In this case we have $H=\mathrm{C}_{2 m+1}, K=\mathrm{D}_{(2 m+1) l}$ and $\Gamma=\mathrm{D}_{l}=\langle g, t\rangle$, where $g$ is the image of $\boldsymbol{\zeta}$ and $t$ is the image of $\mathbf{k}$. The element $g$ acts trivially on $\operatorname{Irr}\left(\mathrm{C}_{2 m+1}\right)$ and ${ }^{t} \rho_{i}=\rho_{2 m+1-i}$. Therefore $\operatorname{Stab}_{\mathrm{D}_{l}}\left(\rho_{i}\right)=\mathrm{C}_{2 l}$ for all $1 \leq i \leq 2 m$. Choose some $1 \leq i \leq 2 m$ and let $p, q \in \mathbf{P}_{i}$ be the two points whose stabilizer is $\mathrm{C}_{2 l}$. Then $\left(\mathrm{C}_{2 l}, p ; \mathrm{C}_{2 l}, q\right)$ is a singular subgroup for all $1 \leq i \leq 2 \mathrm{~m}$.

\section{6. (D)}

In this case we have $H=\mathrm{D}_{m}, K=\mathrm{D}_{2 m}$ and $\Gamma=\mathrm{C}_{2}=\langle g\rangle$, where $g$ is the image of $\boldsymbol{\zeta}$. Note that $g\left(\boldsymbol{\zeta}^{2}\right)=\boldsymbol{\zeta}^{2}$ and $g(\mathbf{k})=\boldsymbol{\zeta}^{2} \mathbf{k}$. The element $g$ acts trivially on all irreducible representations of $H$ except for two of the non-trivial one-dimensional representations, which are swapped. Take any $\mathbf{P}^{1}$ that is fixed by $\Gamma$ and let $p, q \in \mathbf{P}^{1}$ be the two points whose stabilizer is $\Gamma$. Then $\left(\mathrm{C}_{2}, p ; \mathrm{C}_{2}, q\right)$ is a singular subgroup of $\Gamma$.

\section{7. (I)}

In this case we have $H=\mathrm{D}_{2}, K=\mathrm{T}$ and $\Gamma=\mathrm{C}_{3}=\langle g\rangle$, where $g$ is the image of $\boldsymbol{\omega}$. Then $g$ permutes cyclically the three non-trivial one dimensional representations of $H$ and fixes the unique irreducible two dimensional representation, in addition to fixing the trivial representation. Let $\mathbf{P}_{2}$ be the projective line labeled by the two-dimensional irreducible representation of $H$ and $p, q \in \mathbf{P}_{2}$ the two points whose stabilizer is $\Gamma$. Then $\left(\mathrm{C}_{3}, p ; \mathrm{C}_{3}, q\right)$ is a singular subgroup of $\Gamma$.

\section{8. $(\mathrm{M})$}

In this case we have $H=\mathrm{T}, K=\mathrm{O}$ and $\Gamma=\mathrm{C}_{2}=\langle g\rangle$, where $g$ is the image of $\frac{1}{\sqrt{2}}(1+\mathbf{i})$. The element $g$ swaps the two non-trivial one dimensional irreducible representations, swaps the two irreducible two dimensional representation that are not isomorphic to the realization of $\mathrm{T}$ in $\mathrm{SL}_{2}(\mathbf{C})$ and fixes all other irreducible representations (i.e. it is the obvious symmetry of the Dynkin diagram coming from taking duals of representations). Therefore if $\mathbf{P}$ is one of the two exceptional components that is labeled by a non-trivial, self-dual irreducible representation of $\mathrm{T}$ then there are exactly two points $p, q$ in $\mathbf{P}$ whose stabilizer is $\mathrm{C}_{2}$. Then $\left(\mathrm{C}_{2}, p ; \mathrm{C}_{2}, q\right)$ is a singular subgroup of $\Gamma$. 


\section{9. (N)}

In this case we have $H=\mathrm{D}_{2}, K=\mathrm{O}$ and $\Gamma=\mathbf{D}_{3}=\langle u, v\rangle$, where $u$ is the image of $\boldsymbol{\omega}$ and $v$ the image of $\frac{1}{\sqrt{2}}(1+\mathbf{i})$ in $\Gamma$. Hence $u^{3}=v^{2}=1$. We label the irreducible representations of $\mathrm{D}_{2}$ so that $\rho_{0}$ is the trivial representation, $\rho_{2}$ is the two dimensional representation and $\rho_{1}, \rho_{3}$ and $\rho_{4}$ are the three non-trivial one-dimensional representations. Then

$$
u \cdot \rho_{1}=\rho_{4}, \quad u \cdot \rho_{4}=\rho_{3}, \quad u \cdot \rho_{3}=\rho_{1},
$$

and $u$ fixes all other representations. Similarly, $v$ swaps $\rho_{3}$ and $\rho_{4}$ and fixes all other representations. Thus, $\operatorname{Stab}_{\Gamma}\left(\rho_{1}\right)=\mathbf{C}_{2}$. The stabilizer of $\mathbf{P}_{1}$ is $\mathbf{C}_{2}=$ $\langle v\rangle$. Let $p_{1}, q_{1} \in \mathbf{P}_{1}$ be the two points whose stabilizer is $\mathbf{C}_{2}$. The group $\alpha\left(\mathrm{C}_{2}\right)$ will also fix one of the extremal vertices $\rho_{1}, \rho_{3}$ or $\rho_{4}$, without loss of generality we assume that it is $\rho_{3}$. The points in $\mathbf{P}_{3}$ whose stabilizer is $\alpha\left(C_{2}\right)$ are $p_{3}, q_{3}$ say. Since both $\mathrm{C}_{2}$ and $\alpha\left(\mathrm{C}_{2}\right)$ fix the central vertex $\rho_{2}$, one of the two points $p_{i}$ or $q_{i}$ must be the intersection point $\mathbf{P}_{2} \cap \mathbf{P}_{i}$. Let's say its $p_{i}$ in both cases. Then there can be no element of $\mathbf{D}_{3}$ that maps $p_{1}$ to $q_{3}$. Thus, $\left(\mathrm{C}_{2}, p_{1} ; \alpha\left(\mathrm{C}_{2}\right), q_{3}\right)$ is a singular subgroup of $\Gamma$.

\subsection{The cases where $H=\mathrm{C}_{2}$}

In the cases $(\mathrm{F}),(\mathrm{J}),(\mathrm{O}),(\mathrm{S})$, and $(\mathrm{T})$ we have $H=\mathrm{C}_{2}$. Therefore we are in the situation described in (5.2). In all these cases the group $K$ contains at least one element of order $\geq 6$. Therefore we may apply Proposition 5.6 to conclude that $(V, \omega, G)$ does not admit a projective symplectic resolution.

\subsection{1 .}

Now, let $G=G(K, H, \alpha)<\mathrm{Sp}_{4}(\mathbf{C})$ be symplectically irreducible. In order for $V / G$ to admit a symplectic resolution, by Verbitsky's theorem, $G$ must be a symplectic reflection group. If $G$ is improper (cf. $\S 4.1$ ), then $G$ is a complex reflection group, and in this case by [2], $V / G$ can admit a symplectic resolution only if $G=K \curlyvee S_{2}$ for $K$ a cyclic Kleinian group, or else $G=G_{4}$. The latter possibility is excluded, however, since as a subgroup of $\mathrm{GL}_{2}(\mathbf{C})$ it is primitive, and hence as a subgroup of $\mathrm{Sp}_{4}(\mathbf{C})$ it is symplectically primitive. Therefore, by [7, Theorem 2.6], $(K, H, \alpha)$ must be on the list in Tables 1 and 2 .

Summarizing the above calculations and applying Theorem 5.4 therefore implies the following result. 
Theorem 6.4. If $G=G(K, H, \alpha)$ is such that $H \neq\{1\}$ and $H \neq K$, then $(V, \omega, G)$ admits a projective symplectic resolution if and only if $G=$ $G\left(\mathrm{D}_{2}, \mathrm{C}_{2}, \mathrm{Id}\right)$.

Note that $G\left(\mathrm{D}_{2}, \mathrm{C}_{2}, \mathrm{Id}\right)$ is the symplectic reflection group $Q_{8} \times_{\mathbf{Z}_{2}} D_{8}$ studied in [3].

\section{The imprimitive groups in dimension $\geq 6$}

7.1.

As noted in the introduction, by [7, Theorem 2.9], the symplectically imprimitive and irreducible symplectic reflection groups in dimensions greater than four are, up to conjugation, of the form $G_{n}(K, H)$ where $K<\mathrm{SL}_{2}(\mathbf{C})$ is a Kleinian group and $H \leq K$ contains the commutator subgroup $[K, K]$.

Lemma 7.1. The symplectic reflection group $G_{3}\left(\mathrm{C}_{2}, \mathrm{D}_{2}\right)$ does not admit a projective symplectic resolution.

Proof. The group $G_{3}\left(\mathrm{C}_{2}, \mathrm{D}_{2}\right) / \mathrm{C}_{2}^{3} \simeq G_{3}\left(\{1\}, \mathrm{D}_{2}\right)$ acts on $\mathrm{Y} \times \mathrm{Y} \times \mathrm{Y}$ where $\mathrm{Y}=T^{*} \mathbf{P}^{1}$ is the minimal resolution of $\mathbf{C}^{2} / \mathrm{C}_{2}$. We prove that $\mathbf{C}^{6} / G_{3}\left(\mathrm{C}_{2}, \mathrm{D}_{2}\right)$ does not admit a projective symplectic resolution by showing that there exists an isolated point $p \in \mathrm{Y}^{3}$ whose stabilizer with respect to $G_{3}\left(\{1\}, \mathbf{D}_{2}\right)$ is non-trivial i.e. there is an affine open subset $U$ of $p$ such that $G_{3}\left(\{1\}, \mathbf{D}_{2}\right)$ acts freely on $U \backslash\{p\}$. Repeating the argument given in the proof of Theorem 5.4 then implies the claim of the lemma.

In order to simplify things, we consider the action of the larger group $G^{\prime}:=\mathbf{D}_{2} \prec S_{2}$ on $\mathrm{Y}^{3}$. As in the proof of Lemma 5.2 , let

$$
\Delta_{1,2}=\bigcup_{h \in \mathbf{D}_{2}}\left\{(h \cdot x, x, y) \in \mathrm{Y}^{3} \mid(x, y) \in \mathrm{Y}^{2}\right\}
$$

and $\Delta=\Delta_{1,2} \cup \Delta_{1,3} \cup \Delta_{2,3}$ (where $\Delta_{i, j}$ is defined in the obvious manner). This is a proper closed subset of $\mathrm{Y}^{3}$. If there exists some $p \in \mathrm{Y}^{3}$ and $g \in$ $G^{\prime} \backslash \mathbf{D}_{2}^{3}$ such that $g \cdot p=p$ then $p \in \Delta$. To get an isolated point we need to consider points in $\mathbf{P}^{1} \times \mathbf{P}^{1} \times \mathbf{P}^{1}$ not contained in $\Delta$. The group $\mathbf{D}_{2} \simeq$ $\mathrm{C}_{2} \times \mathrm{C}_{2}$ acts on $\mathbf{P}^{1}$ by the image of its reflection representation in $P S L_{2}(\mathbf{C})$. Thus, the three non-trivial elements of $\mathbf{D}_{2}$ are

$$
g=\left(\begin{array}{cc}
i & 0 \\
0 & -i
\end{array}\right), \quad h=\left(\begin{array}{cc}
0 & 1 \\
-1 & 0
\end{array}\right), \quad g h=\left(\begin{array}{cc}
0 & i \\
i & 0
\end{array}\right) \in P S L_{2}(\mathbf{C})
$$


The fixed points of $g$ are $F_{g}=\{[1: 0],[0: 1]\}$, of $h$ are $F_{h}=\{[1: i],[1:-i]\}$ and of $g h$ are $F_{g h}=\{[1: 1],[1:-1]\}$. Each set $F_{w}$ is stable under the action of $\mathbf{D}_{2}$ with both the non-trivial elements of $\mathbf{D}_{2}$ not equal to $w$ swapping the two points in $F_{w}$. If we take

$$
p=([1: 0],[1: i],[1: 1]) \in \mathrm{Y}^{3} \backslash \Delta
$$

then, noting that $g \cdot h \cdot g h=1$ in $P S L_{2}(\mathbf{C})$, the stabilizer of $p$ in $G^{\prime}$ is $\{1, g\} \times\{1, h\} \times\{1, g h\} \simeq \mathrm{C}_{2}^{3}$ and hence the stabilizer of $p$ in $G_{3}\left(\{1\}, \mathbf{D}_{2}\right)$ is $\{(1,1,1),(g, h, g h)\} \simeq \mathrm{C}_{2}$ and $p$ is isolated.

Therefore we may conclude:

Theorem 7.2. Let $n>2$. Then the symplectic quotient $\mathbf{C}^{2 n} / G_{n}(K, H)$ admits a projective symplectic resolution if and only if $K=H$.

Proof. If $G_{n}(K, H) \neq G_{n}\left(\mathrm{C}_{2}, \mathrm{D}_{2}\right)$ and $K \neq H$, then we choose $n-2$ distinct points $p_{3}, \ldots, p_{n}$ in $\mathbf{C}^{2} \backslash\{0\}$ and consider the point $\left(0,0, p_{3}, \ldots, p_{n}\right)$ in $\mathbf{C}^{2 n}$. The stabilizer of this point is $G_{2}(K, H)=G(K, H, \mathrm{Id})$. We have shown in Theorem 6.4 that all those groups $G(K, H, \alpha)$ such that $\Gamma$ is abelian (except for $G\left(\mathrm{D}_{2 l}, \mathrm{C}_{2}, \mathrm{Id}\right)$ ) do not admit projective symplectic resolutions. Now the result follows from [14, Theorem 1.6]. On the other hand, if $G_{n}(K, H)=G_{n}\left(\mathrm{C}_{2}, \mathrm{D}_{2}\right)$ with $n=3$, then we have shown in Lemma 7.1 that the corresponding symplectic quotient does not admit a projective symplectic resolution. If $n>3$ then one can realize $G_{3}\left(C_{2}, D_{2}\right)$ as a parabolic subgroup of $G_{n}\left(\mathrm{C}_{2}, \mathrm{D}_{2}\right)$ as above and the same argument shows that $G_{n}\left(\mathrm{C}_{2}, \mathrm{D}_{2}\right)$ does not admit a projective symplectic resolution.

\section{Proof of the main theorems}

\subsection{Proof of Theorem 1.2}

Assume $G<\mathrm{Sp}_{2 n}(\mathbf{C})$ is symplectically imprimitive and irreducible. As a consequence of Theorems 6.4 and $7.2, \mathbf{C}^{2 n} / G$ cannot admit a projective symplectic resolution if $G=G(K, H, \alpha)$ (for $n=2$ ) or if $G=G_{n}(K, H)$ (for $n>2)$, unless $G=K\left(S_{n}\right.$ or $G=G\left(\mathrm{D}_{2}, \mathrm{C}_{2}, \mathrm{Id}\right) \simeq Q_{8} \times_{\mathbf{z} / 2} D_{8}$. By [7, Theorems 2.2 and 2.9], this includes all cases where $G$ is proper. If $G$ were not proper, then it would be a complex reflection group, and then by [2], $\mathbf{C}^{2 n} / G$ could only admit a projective symplectic resolution if $G$ were a wreath product $(\mathbf{Z} / m)$ ? $S_{n}$ or $G=G_{4}<\mathrm{Sp}_{4}(\mathbf{C})$, although the latter is excluded since it would be symplectically primitive (as $G_{4}<\mathrm{GL}_{2}(\mathbf{C})$ is a primitive complex 
reflection group). Therefore, $\mathbf{C}^{2 n} / G$ can only admit a projective symplectic resolution if it is one of the listed cases. On the other hand, we know that a projective symplectic resolution exists in each of these cases. This completes the proof.

\subsection{Proof of Theorem 1.1}

As before, by [18] we can assume $G$ is a symplectic reflection group, and by [2], we can assume that $G$ is proper. If $G<\mathrm{Sp}_{2}(\mathbf{C})=\mathrm{SL}_{2}(\mathbf{C})$, then we know that a projective symplectic resolution exists. So assume $G<\mathrm{Sp}_{2 n}(\mathbf{C})$ for $n \geq 3$. As explained in $\S 4.1, G$ must be either of the form $G_{n}(K, H)$ or are one of the groups listed in [7, Table III], where $n \leq 5$. In the former case, the result follows from Theorem 1.2. In the latter case, there are seven groups listed, of types $Q, R, S_{1}, S_{2}, S_{3}, T$, and $U$. The table there also lists the stabilizers in each of these groups of roots of the associated quaternionic root system. For each group $G$ and each symplectic reflection $g \in G$, this stabilizer subgroup, call it $H$, is the stabilizer of generic vectors in the image of $g$ - Id. The action of $H$ on the kernel of $g$ - Id identifies $H$ as a subgroup of $\mathrm{Sp}_{2 n-2}(\mathbf{C})$. By [14, Theorem 1.6], if $\mathbf{C}^{2 n} / G$ admits a projective symplectic resolution, so does $\mathbf{C}^{2 n-2} / H$.

In type $Q$, we have $H=G\left(\mathrm{C}_{4}, \mathrm{C}_{2}, 1\right)<\mathrm{Sp}_{4}(\mathbf{C})$, and we showed that $\mathrm{C}^{4} / H$ does not admit a projective symplectic resolution in Theorem 1.2. Similarly, in type $S_{3}$, we have $H=G_{3}\left(\mathrm{D}_{2}, \mathrm{C}_{2}\right)$, and we showed $\mathbf{C}^{6} / H$ does not admit a resolution in the same theorem (or in Lemma 7.1). In type $T$, the group $H$ becomes a complex reflection group, associated to the Coxeter group of type $H_{3}$; in this case $\mathbf{C}^{6} / H$ does not admit a projective symplectic resolution by $[11,12]$ (which is also a special case of the main result of [2] referred to before).

This reduces us to the cases $R, S_{1}, S_{2}$, and $U$, which are the four cases remaining, in dimensions six, eight, eight, and ten respectively.

Remark 8.1. If one could show that type $S_{1}$ does not admit a projective symplectic resolution, then the same would follow for type $U$, since the stabilizer group $H$ mentioned above has type $S_{1}$. Thus, if the four remaining cases (as one might suspect) do not admit projective symplectic resolutions, it suffices only to show it for the three types $R, S_{1}$, and $S_{2}$. 


\section{Questions}

\section{1 .}

By definition, symplectic reflection groups are the symplectic analogue of complex reflection groups. Therefore it is natural to ask which properties of complex reflection groups have natural analogues for symplectic reflection groups. In particular, one can ask if the analogue of Steinberg's Theorem holds:

Question 9.1. Let $(V, \omega, G)$ be a symplectic reflection group, $v \in V$ and $G_{v}=\operatorname{Stab}_{G}(v)$. Let $U$ be the symplectic complement to $V^{G_{v}}$ in $V$. Is $\left(U,\left.\omega\right|_{U}, G_{v}\right)$ a symplectic reflection group?

Remark 9.2. Steinberg's Theorem for complex reflection groups, together with other elementary considerations, show that it suffices to consider the case where $V$ is irreducible as a $G$-module. Furthermore, one can explicitly check for every complex imprimitive group that $\left(U,\left.\omega\right|_{U}, G_{v}\right)$ is indeed a symplectic reflection group. Thus, it actually suffices to resolve the question for the complex primitive, symplectically irreducible symplectic reflection groups; and of these we can further restrict to the case of dimension at least six, since all finite subgroups of $\mathrm{Sp}_{2}(\mathbf{C})$ are symplectic reflection groups. This narrows us down to checking Steinberg's theorem for the seven groups discussed in the previous section. However, if it is indeed the case that the analogue of Steinberg's Theorem holds for symplectic reflection groups, it would be interesting to have a conceptual proof that does not rely on Cohen's classification.

\section{2 .}

To complete the classification of symplectic reflection groups admitting projective symplectic resolutions one needs to answer the following three questions.

Question 9.3. Let $G:=G(K, 1, \alpha) \cong K \rtimes S_{2}$ be a symplectically irreducible proper symplectic reflection group (so that $G$ belongs to one of the families $(G),(K),(P),(Q),(U),(V)$ of Tables 1 and 2). Does the quotient singularity $\mathrm{C}^{4} / G$ admit a projective symplectic resolution? 
Question 9.4. Let $G$ be symplectically primitive and irreducible, but complex imprimitive, i.e., $G<\mathrm{Sp}_{4}(\mathbf{C})$ is one of the groups classified in [7, Lemma 3.3]. Does $\mathbf{C}^{4} / G$ admit a projective symplectic resolution?

Question 9.5. Let $G$ be one of the finitely many primitive exceptional symplectic reflection groups, as listed in [7, Table III]. Does the quotient singularity $V / G$ admit a projective symplectic resolution?

It seems likely that many of these exceptional groups $G$ will contain parabolic subgroups $G_{v}$ such that $\left(U,\left.\omega\right|_{U}, G_{v}\right)$ is known not to admit projective symplectic resolutions. In these cases $(V, \omega, G)$ will also not admit a projective symplectic resolution. In particular, using the stabilizer groups discussed in $§ 8.2$, as we mentioned, this already allows us to eliminate types $Q, S_{3}$, and $T$. Thus there remain at most ten groups in [7, Table III] to check.

\section{Acknowledgments}

The first author is supported by the EPSRC grant EP-H028153. The second author was supported by an AIM five-year fellowship and by NSF grant DMS-0900233. This material is based upon work supported by the NSF under Grant No. 0932078 000, while the authors were in residence at the Mathematical Science Research Institute (MSRI) in Berkeley, California, during 2013. The authors would like to thank Y. Namikawa for patiently answering our questions.

\section{References}

[1] A. Beauville, Symplectic singularities, Invent. Math. 139 (2000), no. 3, $541-549$.

[2] G. Bellamy, On singular Calogero-Moser spaces, Bull. Lond. Math. Soc. 41 (2009), no. 2, 315-326.

[3] G. Bellamy and T. Schedler, A new linear quotient of $\mathbf{C}^{4}$ admitting a symplectic resolution, Math. Zeit. 273 (2013), no. 3-4, 753-769.

[4] J. Bertin, The punctual hilbert scheme: an introduction, in: Geometric Methods in Representation Theory, I, volume 24 of Séminaires et Congrés, pages 1-100. Soc. Math. France, Paris, 2010. 
[5] R. V. Bezrukavnikov and D. B. Kaledin, McKay equivalence for symplectic resolutions of quotient singularities, Tr. Mat. Inst. Steklova 246 (Algebr. Geom. Metody, Svyazi i Prilozh.), 20-42, 2004.

[6] Caucher Birkar, Paolo Cascini, Christopher D. Hacon, and James McKernan, Existence of minimal models for varieties of log general type, J. Amer. Math. Soc. 23 (2010), no. 2, 405-468.

[7] A. M. Cohen, Finite quaternionic reflection groups, J. Algebra 64 (1980), no. 2, 293-324.

[8] W. Crawley-Boevey, On the exceptional fibres of Kleinian singularities, Amer. J. Math. 122 (2000), no. 5, 1027-1037.

[9] P. Etingof and V. Ginzburg, Symplectic reflection algebras, CalogeroMoser space, and deformed Harish-Chandra homomorphism, Invent. Math. 147 (2002), no. 2, 243-348.

[10] B. Fu, A survey on symplectic singularities and symplectic resolutions, Ann. Math. Blaise Pascal 13 (2006), no. 2, 209-236.

[11] V. Ginzburg and D. Kaledin, Poisson deformations of symplectic quotient singularities, Adv. Math. 186 (2004), no. 1, 1-57.

[12] Iain Gordon, Baby Verma modules for rational Cherednik algebras, Bull. London Math. Soc. 35 (2003), no. 3, 321-336.

[13] Y. Ito and I. Nakamura, Hilbert schemes and simple singularities, in: New trends in algebraic geometry (Warwick, 1996), volume 264 of London Math. Soc. Lecture Note Ser., pages 151-233. Cambridge Univ. Press, Cambridge, 1999.

[14] D. Kaledin, On crepant resolutions of symplectic quotient singularities, Selecta Math. (N.S.) 9 (2003), no. 4, 529-555.

[15] M. Lehn and C. Sorger, A symplectic resolution for the binary tetrahedral group, Séminaires et Congres 25 (2010), 427-433.

[16] H. Nakajima, Lectures on Hilbert Schemes of Points on Surfaces, volume 18 of University Lecture Series. American Mathematical Society, Providence, RI, 1999.

[17] Y. Namikawa, Poisson deformations of affine symplectic varieties, Duke Math. J. 156 (2011), no. 1, 51-85.

[18] M. Verbitsky, Holomorphic symplectic geometry and orbifold singularities, Asian J. Math. 4 (2000), no. 3, 553-563. 
School of Mathematics and Statistics, University Gardens

University of Glasgow, Glasgow, G12 8QW, UK

E-mail address: gwyn.bellamy@glasgow.ac.uk

Department of Mathematics

South Kensington Campus, Imperial College

LONDON, LONDON SW7 2AZ, UK

E-mail address: trasched@gmail.com

RECEIVED April 14, 2014 